\title{
Accurate Trajectory and Orientation of a Motorcycle derived from low-cost Satellite and Inertial Measurement Systems
}

\author{
Adrian Waegli 1, Alain Schorderet 2 , Christophe Prongué2, Jan Skaloud $\mathbf{1}$
}

(1) : École Polytechnique Fédérale de Lausanne,

Switzerland, Geodetic Engineering Laboratory

+41(0)216932755/+41(0)216935740

E-mail : \{adrian.waegli,jan.skaloud\}@epfl.ch
(2) : École Polytechnique Fédérale de Lausanne,

Switzerland, Mechnical Systems Design Laboratory

+41(021) $6932955 /+41(021) 6933553$

E-mail : \{alain.schorderet,christophe.prongue\}@epfl.ch

TOPICS: Differential GPS/MEMS-IMU integration performance, trajectory analysis, lateral slipping of tires, determination of characteristics of tires

\begin{abstract}
Inertially aided satellite positioning can bring its benefits to all disciplines in which detailed knowledge of the trajectory is a prerequisite for improving performance. In motorcycling for instance, the determination of slips of tires requires the determination of the precise trajectory and the orientation of the motorcycle's chassis. The correct exploitation of torque or force sensors as well as studies of the vibratory behavior of pneumatics necessitate the knowledge of the orientation of the sensors. Accurate position and orientation can be obtained by integrating inertial measurement units (IMU) with GPS (Global Positioning System). Unfortunately, the traditional, bulky and expensive high-quality GPS/IMU instrumentation is restricted to few disciplines with higher accuracy demands, while the ergonomic constraints of some sports (e.g. ski racing, motorcycling) urge to use devices based on mono-frequency differential GPS and Micro-Electro-Mechanical System (MEMS) inertial technology. Due to their small size, low cost and power consumption, MEMS sensors are suitable for trajectory analysis in sports where ergonomic aspects play an important role. In this article, an experimental low-cost differential GPS/MEMS-IMU system is applied in motorcycling. The system provides an absolute positional accuracy better than $0.5 \mathrm{~m}$, velocity estimates accurate to $0.2 \mathrm{~m} / \mathrm{s}$ and an orientation accuracy of $1-2^{\circ}$.
\end{abstract}

Key words: DGPS, MEMS-IMU, motor cycling, torque sensors, slip of tires

\section{1- Introduction}

Satellite-based positioning provides highly accurate position and velocity. It has already proven its effectiveness in car racing (How, Pohlman et al. 2002), rowing (Zhang, Grenfell et al. 2003) and Alpine Skiing (Skaloud and Limpach 2003). Combined with inertial navigation systems (INS), acceleration and orientation can be observed additionally. Traditional GPS/INS equipment consisting of dual-frequency GPS receivers and tactical-grade INS provides high accuracies even for large dynamics ( $\mathrm{cm}$ for position, $\mathrm{cm} / \mathrm{s}$ for velocity and $1 / 100^{\circ}$ for orientation). However, it is bulky (a few $\mathrm{kg}$ ) and expensive (> $40^{\prime} 000 €$ ) and is therefore unsuitable for many sports. Recently, smaller and lighter equipment consisting of low-cost MEMS triple axis accelerometer and gyroscopes together with inexpensive L1 GPS receivers were introduced by (Waegli and Skaloud 2007). As it has been shown, the combination of these sensors helps to overcome the lack of continuity in the reception of the GPS signals in obstructed environment and to determine accurately the orientation of the MEMS-IMU sensor.

In this article, the design of the GPS/MEMS-IMU system is described. Then, its performance is illustrated based on an experiment where the system was installed on a motorcycle. In the second part of the paper, applications of motorcycling are highlighted where the use of such a system can bring considerable benefits. Firstly, an example of trajectory analysis is given. Secondly, the computation of the lateral slipping of a motorcycle based on the GPS/MEMS-IMU trajectory is explained. Lastly, it will be shown how GPS/INS derived parameters can be exploited in order to characterize the performance of tires.

\section{2- GPS/MEMS-IMU Integration Performance}

MEMS-IMUs are subject to large random and systematic errors (biases, scale factors, misalignment, and noise) which need to be suppressed in order to provide accurate orientations. For instance, a typical bias of $0.5 \mathrm{~m} / \mathrm{s}^{2}$ of a MEMS accelerometer 
would result in positioning errors of 50m after 10s. Representative MEMS gyroscope biases are up to $1 \%$ s and would lead to orientation errors of $10^{\circ}$ after only 10s. A solution for calibrating these errors consists in the integration of MEMS-IMU with satellite positioning. However, the conventional GPS/INS integration strategies (Titterton and Weston 1997) need to be slightly adapted in order to take into consideration the error characteristics of the MEMS-IMU sensors (Waegli, Skaloud et al. 2007).

A motorbike was equipped with MEMS-IMU (xsens MT-i) which is rigidly fixed to the GPS antenna. A low-cost monofrequency receiver (u-blox AEK4) was used together with a dual-frequency GPS receiver from Javad for reference. The results presented below integrate differential L1 GPS solutions of low-cost receivers at $1 \mathrm{~Hz}$ with the triple-axis accelerometer and gyroscope measurements of the MEMS-IMU which are at $100 \mathrm{~Hz}$.
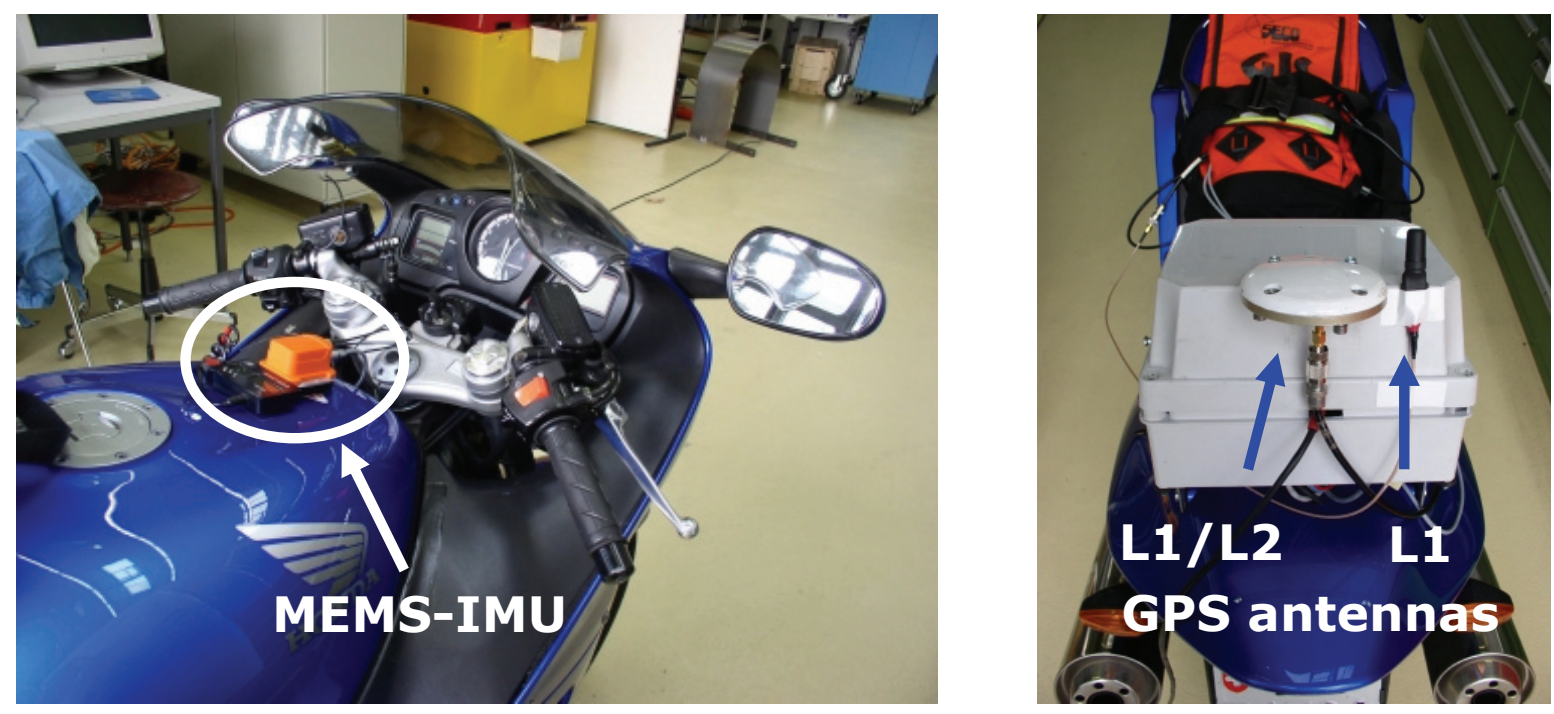

Figure 1 : GPS/MEMS-IMU system setup

The performance of the low-cost L1 GPS/MEMS-IMU system was investigated by (Waegli and Skaloud 2007) in Alpine skiing by comparing its performance to a higher order GPS/INS system. The mean accuracies are summarized in table 1 . The system parameters converge rapidly which permits bridging of GPS data gaps of up to 10s with inertial navigation without loss of accuracy. Using dual-frequency receivers would increase the position accuracy to decimeter-level while velocity and orientation quality would remain at a similar level.

\begin{tabular}{|c|c|c|c|c|c|c|c|c|c|}
\hline & $\mathrm{N}[\mathrm{m}]$ & $\mathrm{E}[\mathrm{m}]$ & $\mathrm{h}[\mathrm{m}]$ & $\mathrm{vN}[\mathrm{m} / \mathrm{s}]$ & $\mathrm{vE}[\mathrm{m} / \mathrm{s}]$ & $\mathrm{vD}[\mathrm{m} / \mathrm{s}]$ & $\mathrm{rl}\left[{ }^{\circ}\right]$ & $\mathrm{pt}\left[{ }^{\circ}\right]$ & $\mathrm{hd}\left[{ }^{\circ}\right]$ \\
\hline RMS & 0.36 & 0.30 & 0.56 & 0.20 & 0.27 & 0.21 & 0.75 & 1.05 & 1.52 \\
\hline
\end{tabular}

Table 1: System setup and mean accuracy of the presented differential GPS/MEMS-IMU system

\section{3- Applications to Motorcycling}

Accurate position, velocity, acceleration and orientation data is crucial in many domains in motorcycling. In the sequel, applications where the GPS/MEMS-IMU system provides innovative approaches are described.

\section{1 - Trajectory Analysis}

The precise trajectory allows visualizing and comparing any parameters related to the performance (Waegli and Skaloud 2007). This may be quantities derived from GPS/MEMS-IMU trajectory (e.g. velocity, acceleration, tire slips) or parameters related to the motorcycle (e.g. throttle, suspensions) or to the athlete (e.g. heart-rate). Figure 6 gives an example where the lateral slipping of the back wheel of the motorbike is visualized on two turns of the track.

\section{2 - Solution to the Reference Frame Problem}

When studying the motorcycle performance, various parameters are determined in different reference frames, e.g. in the reference frame fixed to the motorcycle (abbreviated with $b$ for body) and in the reference frame fixed to the track ( $\ell$ for local-level frame, figure 2). Measurements (e.g. force or torque) can be converted between the two frames thanks to the 
rotation matrix $\mathbf{R}_{b}^{\ell}$ which expresses the orientation of the motorcycle with respect to the track and which is estimated in the GPS/MEMS-IMU integration.

$$
\mathbf{x}^{\ell}=\mathbf{R}_{b}^{\ell} \cdot \mathbf{x}^{b}
$$

where $\mathbf{X}$ stands for observations in either frame.

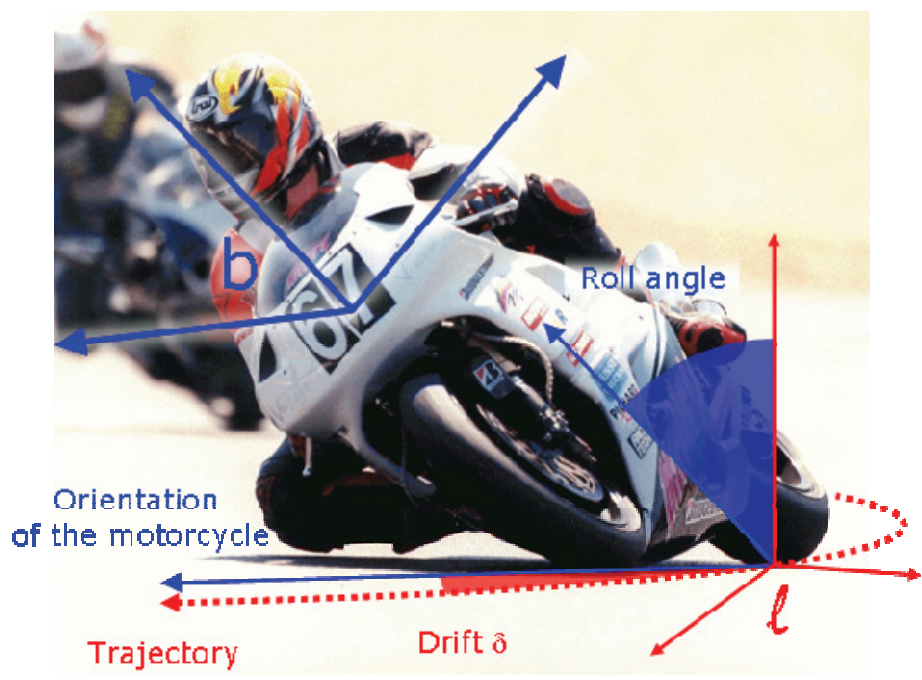

Figure 2 : Illustration of the reference frames

\section{3 - Computation of the Lateral Slipping of Tires}

The lateral slipping of tires can be observed directly with the use of GPS/INS derived results. First, the direction of the trajectory $\alpha$ can be derived from the velocity components $\mathbf{v}_{\text {North }}$ and $\mathbf{v}_{\text {East }}$ (Figure 2) :

$$
\tan (\alpha)=\frac{\mathbf{v}_{\text {North }}}{\mathbf{v}_{\text {East }}}
$$

Furthermore, the GPS/INS integration yields the heading $h d$ of the motorcycle. Combining the two variable leads to the slip of tires $\delta$ :

$$
\delta=h d-\alpha
$$

Figure 3 and figure 4 illustrate the slip angle with respect to the throttle, traction/braking torque at the rear wheel, the roll angle and the lateral acceleration respectively. The confidence level $\left(1 \sigma \approx 1^{\circ}\right)$ highlights the accuracy of the slips. The presented experiment was conducted in winter and the dynamics were correspondingly small. Nevertheless, statistically significant drifts were observed during the turns. The lateral acceleration is consistent with the roll angle. It can be noted that the motorcyclist was inclined even on a great portion of the straight lines (Figure 5). As seen from figure 4, this inclination was compensated by a lateral acceleration. 


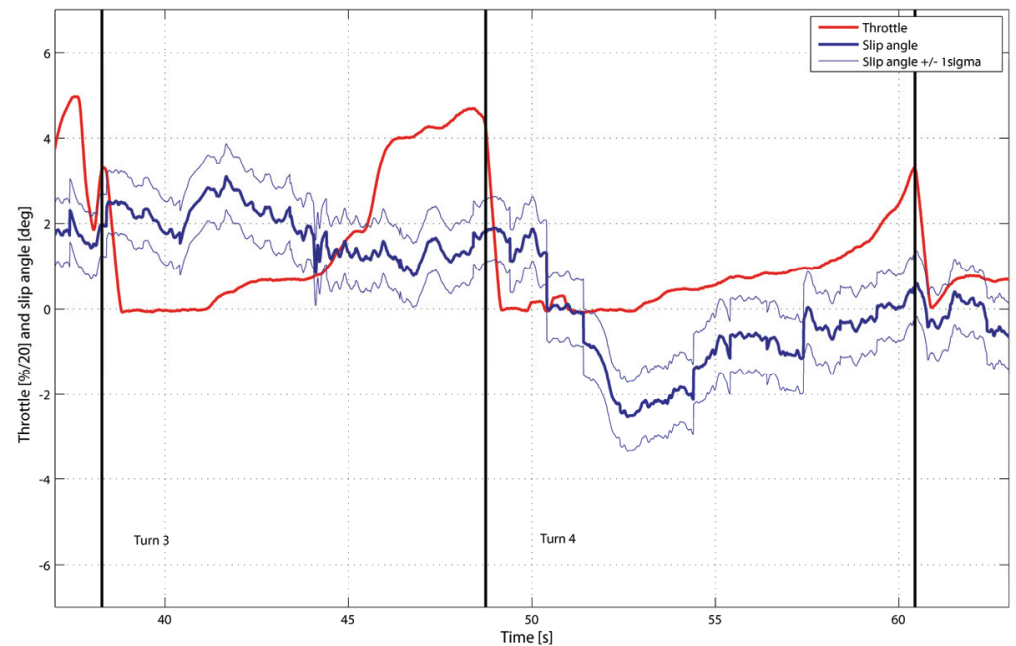

Figure 3 : Throttle with slip angle and accuracy indicator $(1 \sigma)$. The black vertical lines indicate the beginning of the turns.

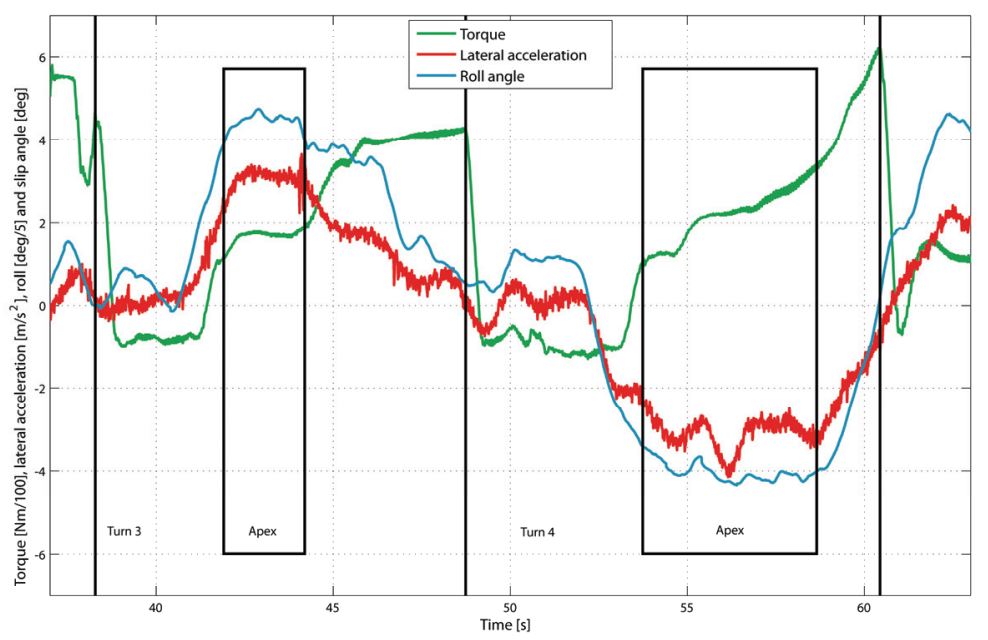

Figure 4 : Torque, lateral accelerations and roll during the same period as figure 3 . The black vertical lines indicate the beginning of the turns.

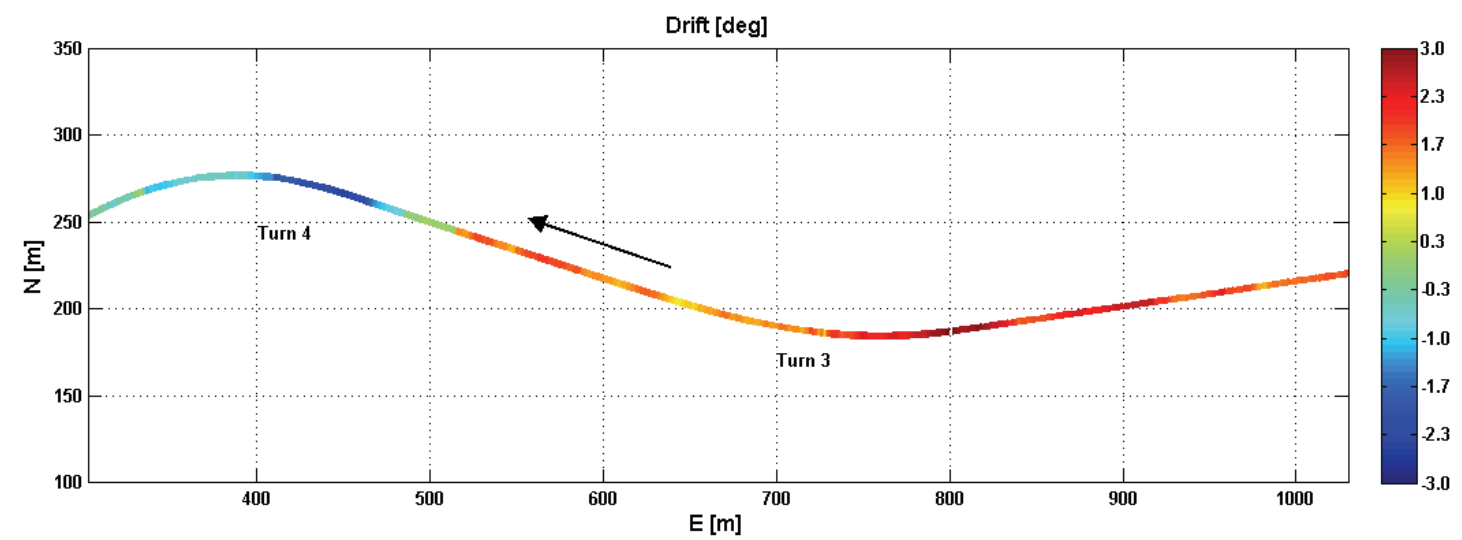

Figure 5 : Lateral slipping angles visualized on the GPS/MEMS-IMU derived trajectory 


\section{4 - Evaluation of the Characteristics and Performance of Tires}

The "magic formula tire model" defined by (Pacejka and Bakker 1992) provides a mathematical expression from which forces and moments acting longitudinally or laterally from the road on the tire can be related to its slip performance:

$$
\begin{aligned}
& y(x)=D \cdot \sin (C \cdot \arctan (B \cdot x-E \cdot(B \cdot x-\arctan (B \cdot x)))) \\
& Y(X)=y(x)+\Delta y \\
& x=X+\Delta x
\end{aligned}
$$

where

- $\quad X$ represents the lateral slipping angle $\delta$ or the longitudinal slip $\kappa$.

- $Y$ stands for the lateral force $F_{y}$, the aligning torque $M_{z}$ or longitudinal force $F_{x}$.

- $\quad B, C, D, E, \Delta x$ and $\Delta y$ are constant coefficients.

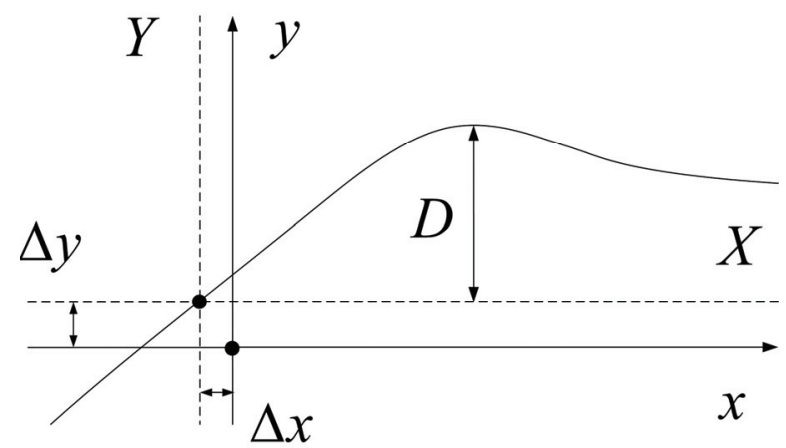

Figure 6 : Typical tire characteristics indicating the meaning of some coefficient of equation 4.

The constant coefficients are usually determined by laboratory experiments (on drum). The GPS/MEMS-IMU system permits the calibration of these deterministic parameters in situ. First, force and torque measurements need to be referenced with respect to the road which becomes possible due to the orientation determined by GPS/INS. The lateral slip angle is directly computed from GPS/INS and needs only to be corrected for the steering of the front wheel, the tire radius variation due to speed, load and roll angle as wells as the suspension pitch which can be measured directly by means of linear potentiometers. The longitudinal slip requires the knowledge of the longitudinal velocity ( $v_{s x}$, from GPS/INS) and of the longitudinal velocity of the tire at the contact patch $\left(v_{x}\right.$, from digital speed sensors, which must be compensated for the tire radius variation ):

$$
\kappa=-\frac{v_{s x}}{v_{x}}
$$

Hence, combining all these measurements permits to characterize tires in the field. This calibration reflects the actual characteristics of the surface (temperature and road roughness) and therefore refines laboratory findings. The peak value of $Y$ is often situated at approximately $10^{\circ}$ of slipping and slipping angles up to $30^{\circ}$ can be modeled. In this range, the accuracy of the slipping angles accounts therefore for $3-10 \%$ of the error, whereas the torque and force measurements can be reached with a level of $5 \%$.

\section{5 - Tire related vibrations}

Attitude determination also provides essential data to the motorcycle vibration analysis. Indeed, tire-related vibrations are today very important in motorcycle racing. The so-called "chattering" vibrations may appear at the curves" entry, apex or exit and enforce the rider to reduce its speed. To solve this problem, the phenomenon is studied by means of numerical and experimental approaches (Duvernier, Fraysse et al. 2002). One approach consists in using hybrid eigenvector bases to perform a modal synthesis (Schorderet and Gmür 2004) (Schorderet 1997). The vibratory behavior of a given tire is depending on two external parameters: ground load (vertical) and roll angle. To build the modal basis, a laboratory experimental modal analysis provides the eigenfrequencies and eigenmodes for discrete values of these parameters. Then, 
vibration measurements are realized on the track and dedicated software is applied to identify the frequencies where chattering exists (rider data). The hybrid model is then used to reduce or cancel the chattering vibrations.

The efficiency of this predictive model is depending on the experimental data quality. The use of the low-cost L1 GPS/MEMS-IMU system combined with a force measurement unit (body reference frame) allows determining accurate roll angles and evaluating the vertical force in the local reference frame. These two parameters are required for the definition of the precise dynamic conditions under which the vibrations appear.

\section{4- Conclusion}

A low-cost GPS/MEMS-IMU system was presented which is suitable in terms of cost and ergonomy for deployment in motorcycling. The system continuously tracks the 3D-trajectory of the motorcycle which allows monitoring and comparing a large number of parameters related to the performance. Any performance parameter can be represented with respect to the trajectory and compared to subsequent turns or laps. Its accuracy $\left(50 \mathrm{~cm}\right.$ in position, $<0.2 \mathrm{~m} / \mathrm{s}$ for velocity and $1-2^{\circ}$ for orientation) opens new possibilities in analyzing many factors related to motorcycle performance.

The system provides orientations which enable the computation of the lateral slipping angles in relation to the track and measurements of the motorcycle (e.g. force, torque measurements). Combining all this data enables the calibration of tire models in situ.

\section{5- Acknowledgement}

This research is financed by TracEdge, based at Grenoble, France. The experimental tests have been performed using torque sensors provided by NRCtech, based at EPFL Science Park, Lausanne, Switzerland.

\section{6- References}

Duvernier, M., P. Fraysse, et al. (2002). Tyre Modelling for NVH Engineering in ADAMS. 1st MSC.ADAMS European User Conference, London.

How, J., N. Pohlman, et al. (2002). GPS Estimation Algorithms for Precise Velocity, Slip and Race-track Position Measurements. SAE Motorsports Engineering Conference \& Exhibition.

Pacejka, H. B. and E. Bakker (1992). "The magic formula tyre model." Vehicle System Dynamics - International Journal of Vehicle Mechanics and Mobility 21: 1-18.

Schorderet, A. (1997). Synthèse modale et problème inverse en dynamique des structures. Lausanne, EPFL. PhD: 1698.

Schorderet, A. and T. Gmür (2004). "Structural dynamics optimization based on a hybrid inverse synthesis method using a quadratic approximation." ASME Transactions : Journal of Vibration and Acoustics 126(2): 253-259.

Skaloud, J. and P. Limpach (2003). Synergy of CP-DGPS, Accelerometry and Magnetic Sensors for Precise Trajectography in Ski Racing. ION GPS/GNSS 2003, Portland.

Titterton, D. H. and J. L. Weston (1997). Strapdown inertial navigation technology, Peter Peregrinus Ltd.

Waegli, A. and J. Skaloud (2007). Assessment of GPS/MEMS-IMU Integration Performance in Ski Racing. ENC, Geneva, Switzerland.

Waegli, A. and J. Skaloud (2007). "Turning Point - Trajectory Analysis for Skiers." InsideGNSS(Spring 2007).

Waegli, A., J. Skaloud, et al. (2007). Assessment of the Integration Strategy between GPS and Body-Worn MEMS Sensors with Application to Sports. ION GNSS, Fort Worth, Texas.

Zhang, K., R. Grenfell, et al. (2003). Towards a Low-Cost, High Output Rate, Real-Time GPS Rowing Coaching and Training System. 16th International Technical Meeting of the Satellite Division of The Institute of Navigation, Portland. 